ECONOMÍA: TEORÍA Y PRÁCTICA • Nueva Época, número 44, enero-junio 2016, pp. 115-146, http://www.izt.uam.mx/economiatyp/ojs

\title{
Estrategias dinámicas de cobertura cruzada eficiente para el mercado del petróleo mexicano: \\ Evidencia de dos modelos GARCH multivariados con término de corrección de error*
}

\section{Dynamic Strategies of Efficient Cross Hedging for the Mexican Oil Market: \\ Evidence from Two GaRCH Multivariate Models with Error Correction Term}

Raúl de Jesús Gutiérrez**

\section{RESUMEN}

Este trabajo amplía los modelos de correlación condicional dinámica de Engle y de Tse y Tsui al incorporar términos de corrección de error en el diseño de estrategias de cobertura cruzada dinámicas de varianza mínima para el petróleo mexicano. Respecto a la reducción del riesgo, la evidencia empírica confirma el desempeño superior del modelo MGARCH-CCD de Engle cuando se utiliza el mercado de futuros del WTI como mecanismo de cobertura, en particular para los crudos Olmeca e Istmo. Los hallazgos tienen importantes implicaciones económicos-financieras para gobierno y consumidores, debido a la eficiencia y transparencia de las coberturas cruzadas implementadas para reducir el riesgo de bajos precios.

Palabras clave: modelos MGARCH-CCD con término de corrección de error, razón de cobertura cruzada óptima, índice eficiente de cobertura, mercados de futuros petroleros.

Clasificación JEL: C22, C32, G11, G17, G32.

\begin{abstract}
This article extends the dynamic conditional correlation models of Engle and Tse and Tsui by incorporating error correction terms in order to devise dynamic minimum variance crosshedging strategies for Mexican crude oil. In terms of out-of-sample risk reduction, the empirical evidence confirms the superior performance of Engle's DCC-MGARCH model when the WTI crude oil futures market is used as a hedging mechanism, especially for Olmeca and Istmo crude oils. The findings have a number of important economic-financial implications for government and consumers because of the effectiveness and transparency of the cross hedging implemented to reduce the risk of lower transaction costs in the Mexican crude oil.

Keywords: error correction DCC-MGARCH models, optimal cross-hedging ratio, hedging effectiveness index, crude oil futures markets. JEL classification: C22, C32, G11, G17, G32.

* Fecha de recepción: 14/07/2014. Fecha de aprobación: 19/02/2016.

** Departamento de Actuaría de la Facultad de Economía de la Universidad Autónoma del Estado de México. Correo electrónico: rjg2005mx@yahoo.com.mx.
\end{abstract}




\section{INTRODUCCIÓN}

El nuevo milenio ha sido testigo de importantes e inesperados cambios en lo económico y lo financiero en el mercado de materias primas, en particular, el petróleo. El sólido y constante crecimiento económico global alimentado por el creciente consumo de energía fósil, principalmente por las economías emergentes de China y la India, ha contribuido al rápido desarrollo del mercado del petróleo, al grado de consolidarlo en un mercado financiero sofisticado y líquido. Sin embargo, factores económico-financieros, sociopolíticos, geopolíticos, tecnológicos e, incluso, de carácter climático, así como de capacidad de refinamiento, de reservas y de acuerdos institucionales internacionales, lo han transformado en una plataforma más volátil y riesgosa, a diferencia de otras materias primas y/o mercados accionarios y cambiarios. ${ }^{1}$

La elevada volatilidad generada por las fluctuaciones extremas en los precios del petróleo afecta directamente la distribución del ingreso de productores, consumidores, compañías y refinadoras de petróleo. Este fenómeno característico del mercado no se puede controlar con el simple uso de las reservas de petróleo, además de que su sustitución por una fuente de energía alternativa es complicada en el corto plazo. En consecuencia, el mercado de futuros desempeña un importante papel como mecanismo en la prevención del riesgo de precios adversos. El diseño de estrategias de cobertura con contratos de futuros ${ }^{2}$ permite compensar las pérdidas generadas por los movimientos desfavorables en los precios al contado. Por otra parte, las operaciones de posiciones cortas y largas en los mercados de futuros tienen el potencial para reducir factores de riesgo como la falta de liquidez y el incumplimiento.

Desde que el grado de incertidumbre en los mercados del petróleo se incrementó, a raíz de la liberalización del sector energético en la segunda mitad de los años ochenta, se han implementado mercados de futuros de crudo ligero para administrar apropiadamente la exposición al riesgo de altibajos en los precios a

${ }^{1}$ Motivados por la fuerte alza en los precios del petróleo y la correlación negativa entre los mercados de energía y accionarios, los inversionistas institucionales, como fondos de pensiones y fondos de inversión de riesgo moderado y alto (hedge funds), han incorporado contratos de futuros sobre petróleo en sus portafolios de títulos de capital como mecanismo para la diversificación del riesgo (Geman y Kharoubi, 2008).

${ }^{2}$ Un contrato de futuros es un acuerdo privado entre dos partes, en donde se fija la cantidad, la calidad y el precio de una materia prima que se va a liquidar en una fecha futura y entregar en un lugar establecido. 
través de estrategias de cobertura óptima. De hecho, la literatura ha demostrado que tales estrategias reducen la volatilidad en los precios del petróleo sin afectar los rendimientos y con la ventaja adicional de incrementar su efectividad con un mayor grado de certeza (Daniel, 2001). Básicamente, los contratos de futuros, que otorgan cobertura para fijar el precio físico del petróleo, se cotizan en la New York Mercantile Exchange (NYMEX) y en la Intercontinental Exchange (ICE) de Londres. Sus principales marcadores de referencia son los crudos ligeros West Texas Intermediate (WTI), de Estados Unidos, y Brent, del mar del Norte.

Desafortunadamente, la variedad de petróleos extraídos de diferentes zonas geográficas (en más de cien países) ha limitado la presencia de productores, consumidores y refinerías en los mercados de futuros internacionales. Por ello, su volumen de operación para la transferencia del riesgo se reduce notablemente como consecuencia de la creciente comercialización de desiguales niveles de calidad. En este sentido, el problema de la cobertura óptima ha dado lugar a la creación de una serie de innovaciones financieras para la administración del riesgo. Esto es, transacciones bilaterales que se llevan a cabo en mercados no regulados u over the counter (ОTC). Las operaciones más comunes vía contratos ОTC sobre petróleo son las permutas financieras (swaps) y las opciones financieras. Pese a su flexibilidad y diseño, hecho a la medida de los clientes, esta clase de coberturas son más riesgosas y costosas, debido al peligro de incumplimiento de las contrapartes, a diferencia de los mercados de futuros, que cuentan con estructuras y procedimientos con mayor transparencia para la contratación, compensación y liquidación.

En el contexto de las estrategias de cobertura, los países productores y exportadores de petróleo, como México, resienten la falta de un mercado de futuros para la protección directa del volumen de producción. Con el fin de mitigar las severas caídas en los precios de referencia internacional, la Secretaría de Hacienda y Crédito Público (SHCP) mexicana vende contratos de opciones OTC a través de bancos de inversión e intermediarios de hidrocarburos. Sin embargo, las operaciones de cobertura, creadas por los diseñadores de la política energética y fiscal, han sido un total fracaso en los últimos años, puesto que el costo del seguro petrolero ha representado un elevado gasto en la protección del Presupuesto de Egresos de la Federación.

A finales de diciembre de 2012, el costo de la prima del seguro se estableció en USD 904.1 millones, aproximadamente. Pero las estrategias llevadas a cabo para proteger 1183 millones de barriles diarios de las fluctuaciones extremas en el precio internacional del petróleo no tuvieron los resultados esperados, 
debido al hecho de que el precio de la mezcla mexicana de exportación se aseguró en USD 86 por barril, cuando su rango promedio fue de USD 99 y su máximo, de USD 112 durante 2013. Además, pese a que registró un mínimo de USD 88.58, se mantuvo por arriba del precio fijado por las autoridades gubernamentales. Es importante resaltar que este tipo de coberturas sólo ha tenido éxito en 2009, cuando el gobierno obtuvo beneficios por el ejercicio de la opción de venta ( $p u t)$, pero con una prima muy alta que alcanzó un valor aproximado de USD 1500 millones.

Debido a los resultados negativos de los programas de cobertura petrolera llevados a cabo en los mercados ОтС internacionales con contratos de opciones financieras, así como al hecho de que existe una correlación positiva y un alto grado de integración entre los petróleos nacionales y el wTI y el Brent en los recientes años, ${ }^{3}$ la SHCP debe comenzar a desarrollar estrategias de cobertura eficientes, usando contratos de futuros sobre los petróleos de referencia. Este mecanismo permitirá controlar apropiadamente el impacto de los riesgos de mercado en los ingresos petroleros del país, originado por los efectos adversos de la volatilidad en los mercados internacionales. Además, las operaciones serán más transparentes y con costos de transacción relativamente más bajos, evitando con ello recortes en el presupuesto del gasto público que afecten la inversión en infraestructura productiva y social, y salvaguardando el impulso a la competitividad de la economía y el incremento del nivel de vida de las familias mexicanas. De la misma manera, los consumidores industriales pueden beneficiarse de las estrategias de cobertura cruzada para amortiguar los impactos negativos en los precios del petróleo nacional, al participar en los mercados de futuros internacionales con posiciones opuestas.

El objetivo del presente trabajo es probar si las estrategias de cobertura cruzada diseñadas a través de contratos de futuros sobre petróleo de referencia internacional permiten mitigar la volatilidad del precio del petróleo mexicano. Para ello, se estima la razón de cobertura óptima y se evalúa la eficiencia de la cobertura cruzada con datos diarios de los precios físicos de los crudos Maya, Istmo y Olmeca, y los precios de los futuros del wTI y el Brent en el periodo del 2 enero de 2000 al 31 de diciembre de 2013.

En numerosos análisis sobre la estimación de la razón de cobertura óptima, se ha empleado la tradicional reversión por mínimos cuadrados ordinarios (MCO) de los rendimientos de los precios de contado respecto a los rendimientos

\footnotetext{
${ }^{3}$ Para un análisis más profundo del grado de integración entre los mercados internacionales y nacionales del petróleo, véase, por ejemplo, De Jesús (2014).
} 
de los futuros. Esta técnica asume que la volatilidad tiene un comportamiento constante a través del tiempo (Ederington, 1979; Figlewski, 1984; Myers y Thompson, 1989; Benet, 1992). No obstante, las estrategias de cobertura basadas en modelos estáticos pueden ser contraproducentes para neutralizar el riesgo de precios volátiles. Estudios empíricos sustentan que la dinámica de la varianza, covarianza y correlaciones cambia con el tiempo y es altamente persistente en las variables financieras, especialmente el petróleo mexicano (Dávila, Núñez y Ruiz, 2006; Lorenzo, Durán y Armenta, 2012; De Jesús y Carvajal, 2013). Para mejorar la eficiencia de las coberturas bajo diferentes condiciones de mercado, los modelos MGARCH (multivariated generalized autoregressive conditional heteroskedasticity) son excelentes alternativas para recoger los segundos momentos condicionales de la distribución conjunta y estimar la razón de cobertura óptima (Cecchetti, Cumby y Figlewski, 1988; Baillie y Myers, 1991; Kroner y Sultan, 1993; Park y Switzer, 1995; Moschini y Myers, 2002; Haigh y Holt, 2002; Choudhry, 2009).

Sin embargo, la mayoría de la literatura mencionada se enfoca en la estimación de la razón de cobertura óptima y la eficiencia de las estrategias de cobertura para información dentro de la muestra. En este sentido, la primera contribución de este trabajo es desarrollar estrategias de cobertura cruzada de mínima varianza fuera de la muestra. Por lo tanto, los modelos MGARCH de correlación condicional dinámicos (MGARCH-CCD) de Engle (2002) y de Tse y Tsui (2002) se reestiman a través de una venta móvil, la cual remueve la observación más remota y agrega la más reciente al periodo de muestra. Este procedimiento captura la información más nueva del mercado y actualiza las estrategias de cobertura cruzada óptima. De esta manera, la exposición al riesgo de precios volátiles de los crudos nacionales se transfiere de manera dinámica a través de contratos de futuros sobre petróleo wTI y Brent.

Por otra parte, pocos estudios han incorporado en sus modelos el concepto de cointegración, particularmente para petróleos de baja densidad y alto contenido de azufre, lo que también es conocido en la literatura como tendencia estocástica o relación de equilibrio de largo plazo entre los mercados de contado y futuros. Una segunda contribución es agregar el término de corrección de error a las ecuaciones de las medias condicionales de los modelos MGARCH-CCD. Esta variable controla las desviaciones de la relación de equilibrio de largo plazo en un periodo, lo que se espera sea corregido en el siguiente periodo. Probablemente, la omisión del término de corrección de error puede tener serias implicaciones económico-financieras en la predicción de las razones de cobertura óptima y 
desempeño de las coberturas cruzadas, dado que la literatura ha sustentado que los cambios en los precios de contado y futuros comparten la misma tendencia estocástica en el largo plazo bajo supuestos débiles (Toyoshima, Nakaijima y Hamori, 2013). Finalmente, el estudio también hace contribuciones a la literatura empírica de la administración de riesgos y a la literatura econométrica.

La exposición se estructura de la siguiente forma: en la sección I se discute la literatura empírica revisada, mientras que la in presenta las estrategias de cobertura y amplía los modelos MGARCH-CCD incorporando el término de corrección de error. En la sección III se realiza un análisis preliminar de los datos utilizados a través de las estadísticas básicas, pruebas de raíz unitarias y de cointegración, y se discuten los resultados de las coberturas cruzadas estimadas de los modelos MGARCH-CCD. Finalmente, se plantean las principales conclusiones.

\section{LITERATURA RELACIONADA}

En la literatura que trata el tema de la cobertura utilizando los mercados de futuros como mecanismo para transferir y reducir la exposición al riesgo, la mayoría de los análisis se basan principalmente en los índices bursátiles y las divisas. Sin embargo, existen pocos estudios que proporcionen evidencia para los mercados de materias primas, en particular el petróleo, a pesar de que su volumen de operación se ha incrementado en los últimos años.

Dentro de la literatura que estudia la relación entre los precios forward y los futuros, Lanza, Manera y McAleer (2006) estiman las correlaciones de los rendimientos del WTI a través del modelo de correlación condicional dinámico (CCD). La evidencia empírica rechaza el supuesto de correlación constante. Aplicando varios modelos de volatilidad condicional multivariados a datos del petróleo Tapis, de Malasia, ${ }^{4}$ Manera, McAleer y Grasso (2006) mostraron la importancia de la correlaciones dinámicas para explicar si los rendimientos de los precios de contado y forward se sustituyen o complementan, así como su uso fundamental en el diseño de estrategias de cobertura y valuación de productos derivados. Utilizando datos diarios de los precios de contado -y sus respectivos contratos forward y futuros sobre marcadores wTI, Brent y Dubái-, Chang, McAleer y Tansuchat (2009) evidencian que las CCD juegan un papel importante en el proceso de la diversificación del riesgo en los mercados internacionales del petróleo.

\footnotetext{
${ }^{4}$ Pese a que no se cotiza en un mercado líquido, como en los casos del wTI y del Brent, el Tapis se usa como marcador de referencia para la fijación de los precios en los mercados de Asia y Australia.
} 
Entre los estudios sobre la razón de cobertura óptima, Haigh y Holt (2002) fueron los pioneros en utilizar metodologías MGARCH para cubrir la incertidumbre en el precio futuro de los energéticos. La evaluación del análisis dentro y fuera de la muestra revela que la reparametrización mediante el modelo BEKK (Baba-Engle-Kraft-Kroner) proporciona el mejor desempeño predictivo, en cuanto a la reducción del riesgo para los comerciantes de energía cuando se toman posiciones de compra-venta simultáneas en contratos de futuros sobre petróleo y sus productos refinados, como gasolina sin plomo y combustible para calefacción. Utilizando diferentes tipos de petróleo y varios contratos de futuros listados en las bolsas de Nueva York y Londres, Alizadeh, Kavussanosy Menachof (2004) estudian la eficiencia de las coberturas cruzadas dentro y fuera de la muestra. El control de los efectos desfavorables en los precios de los combustibles líquidos, en barcos de los puertos de Rotterdam, Singapur y Houston, es mejor a través del modelo de vectores de corrección de error multivariado con especificación de residuos GARCH, aunque los hallazgos indican que la administración del riesgo en la industria del combustible líquido es realmente limitada.

Por otra parte, Jalali y Kazemi (2006) aplican modelos MGARCH a datos semanales de los precios físicos y futuros del WTI para los diferentes vencimientos listados en la NYMEX. Los resultados del modelo BEKK ${ }^{5}$ confirman que las razones de cobertura óptima tienden a cambiar con el tiempo y se incrementan con la duración del contrato, en particular para el vencimiento de cuatro meses. Asimismo, Ripple y Moosa (2006) estudian el efecto del vencimiento de los contratos de futuros en la eficiencia de las operaciones de cobertura, utilizando datos diarios y mensuales para el periodo de enero de 1998 a abril de 2005. Sus resultados muestran que las estrategias de cobertura son más efectivas y menos costosas cuando los inversionistas se protegen con el contrato de futuros más próximo a vencer.

En estudios más recientes, Chang, McAleer y Tansuchat (2010) estiman tres modelos MGARCH para transferir el riesgo de altibajos en los precios de los principales mercados internacionales del petróleo, al diseñar estrategias de cobertura convencionales y cruzadas con forward y futuros. El estudio muestra que la razón de cobertura óptima es relativamente alta en relación con los petróleos dulces y ligeros (WTI y Brent), independientemente del mercado que se utilice para ella. En contraste, los petróleos ácidos y pesados (Dubái y Tapis) tienen razones de cobertura óptima pequeñas, especialmente la relación Tapis-WTI del

\footnotetext{
${ }^{5}$ Para más detalles técnicos de la estructura MGARCH, véase Engle y Kroner (1995).
} 
mercado forward. Usando datos diarios de los precios de contado y futuros del WTI y el Brent, Chang, McAleer y Tansuchat (2011) aplican varias especificaciones MGARCH para el análisis de las razones de cobertura óptima y su eficiencia. Los resultados empíricos resaltan, en términos de la reducción de la varianza del portafolio, el potencial del modelo BEKK diagonal y el pobre desempeño de la aproximación BEKK en la estimación de la razón de cobertura óptima.

Hung et al. (2011) proponen un modelo bivariado con cuatro cambios de régimen y estiman las razones de cobertura de mínima varianza del petróleo WTI. Su desempeño dentro y fuera de la muestra es superior a varios modelos de volatilidad multivariados, excepto cuando la evaluación se realiza con la prueba estadística de capacidad superior de predicción. ${ }^{6}$ En un estudio que discute las estrategias de cobertura cruzada entre mercados de petróleo y sus productos refinados, Wang y Wu (2012) encontraron evidencia empírica de que el modelo BEKK escalar es la mejor opción para cubrir la incertidumbre del WTI a través de los mercados de contado de la gasolina y turbosina, mientras que el combustible para calefacción es la mejor cobertura con el modelo BEKK diagonal. Assis (2013) compara varios modelos clásicos para evaluar la eficiencia de las estrategias de cobertura, y encuentra que los modelos vech diagonal y de vectores autorregresivos proporcionan inmejorables razones de cobertura para prevenir riesgos en el caso del WTI. Asimismo, Toyoshima, Nakaijima y Hamori (2013) proporcionan nueva evidencia de la efectividad de los modelos de CCD simétrico y asimétrico en la formulación de estrategias de cobertura para transferir la exposición al riesgo en el precio del WTI.

\section{ESTRATEgias de COBERTURA y METOdología}

En esta sección se describen las estrategias de cobertura cruzada para el petróleo nacional con posiciones de futuros de los principales mercados de referencia internacional y los modelos de volatilidad multivariados.

\section{Razón de cobertura cruzada óptima}

La razón de cobertura tiene como objetivo minimizar la varianza de una posición de contado abierta. En este tenor, la estrategia naive es la forma más sencilla para

\footnotetext{
${ }^{6}$ Para una descripción técnica más detallada de la prueba de capacidad superior de predicción, véase Hansen (2005).
} 
cubrir el riesgo al incluir contratos de futuros en el portafolio de inversión. Esta estrategia consiste en vender (corta) o comprar (larga) una unidad de contratos de futuros equivalente en magnitud, pero con signo opuesto a la posición de contado. La principal desventaja de esta cobertura se atribuye a que no toma en cuenta el riesgo de base, también conocido como correlación imperfecta entre cambios en los precios de futuros y cambios en los precios de contado. Además, si la base varía con el tiempo, la cobertura óptima de la posición será imposible con la estrategia naive.

En general, los participantes en los mercados de futuros diseñan estrategias de cobertura de acuerdo a sus expectativas de mercado y sus objetivos de inversión. En el caso de los productores, como México, o de consumidores que desean cubrir con coberturas cruzadas su exposición a las fluctuaciones en los precios del petróleo, los rendimientos esperados de los portafolios de cobertura formados por posiciones de contado y futuros pueden definirse como

$$
\begin{aligned}
& \mathrm{E}\left[R_{C, t}\right]=\mathrm{E}\left[R_{S, t}\right]-h_{t} \mathrm{E}\left[R_{F, t}\right] \\
& \mathrm{E}\left[R_{C, t}\right]=-\mathrm{E}\left[R_{S, t}\right]+h_{t} \mathrm{E}\left[R_{F, t}\right],
\end{aligned}
$$

donde $R_{C, t}$ indica el rendimiento del portafolio de cobertura entre $t-1 \mathrm{y} t . R_{S, t}$ y $R_{F, t}$ indican los rendimientos de las posiciones de contado y de futuros entre $t-1$ y $t . h_{t}$ es la razón de cobertura o número de futuros requeridos para cubrir la posición de contado abierta. Para la identificación de posibles diferencias en las volatilidades de los mercados de contado y de futuros es necesario minimizar la varianza del portafolio de cobertura. En la teoría de la cobertura se define de la siguiente forma:

$$
\operatorname{Var}\left[R_{C, t} \mid I_{t-1}\right]=\operatorname{Var}\left[R_{S, t} \mid I_{t-1}\right]-2 h_{t} \operatorname{Cov}\left[R_{S, t}, R_{F, t} \mid I_{t-1}\right]+h_{t}^{2} \operatorname{Var}\left[R_{F, t}\right]
$$

donde $I_{t-1}$ es el conjunto de información disponible en el periodo $t-1$. $\operatorname{Var}\left[R_{S, t} \mid I_{t-1}\right], \operatorname{Var}\left[R_{F, t} \mid I_{t-1}\right]$ y $\operatorname{Cov}\left[R_{S, t}, R_{F, t} \mid I_{t-1}\right]$ son las varianzas y covarianzas condicionales de los rendimientos de las posiciones de contado y de futuros, respectivamente.

La razón de cobertura óptima $h_{t}$ se obtiene optimizando la varianza condicional del portafolio de cobertura, es decir, $\operatorname{Min}_{h_{t}}\left[\operatorname{Var}\left[R_{C, t} \mid I_{t-1}\right]\right]$. De esta manera, calculando la derivada parcial de la ecuación (3) con respecto a $h_{t}$, e 
igualando a cero, la razón de cobertura óptima condicional sobre la información disponible en el periodo $t-1$ es igual a

$$
h_{t}^{*} \mid I_{t-1}=\frac{\operatorname{Cov}\left[R_{S, t}, R_{F, t} \mid I_{t-1}\right]}{\operatorname{Var}\left[R_{F, t} \mid I_{t-1}\right]} .
$$

La razón de cobertura de mínima varianza es la proporción de la covarianza entre los rendimientos de las posiciones de contado y de futuros y la varianza de los rendimientos de la posición de futuros. Frecuentemente, el valor de la razón de cobertura es menor a la unidad porque minimiza el riesgo ante la presencia del riesgo de base.

En la evaluación de la razón de cobertura óptima fuera de la muestra, $\mathrm{Ku}$, Chen y Chen (2007) proponen el índice eficiente de cobertura (ICE). Este indicador reduce la varianza del portafolio de cobertura cuando la comparan con la varianza del portafolio sin cobertura. Esta medida convencional se puede expresar de la siguiente manera:

$$
I C E=\left[\frac{\operatorname{Var}\left[R_{S, t} \mid I_{t-1}\right]-\operatorname{Var}\left[R_{C, t} \mid I_{t-1}\right]}{\operatorname{Var}\left[R_{S, t} \mid I_{t-1}\right]}\right],
$$

donde $\operatorname{Var}\left[R_{C, t} \mid I_{t-1}\right]$ y $\operatorname{Var}\left[R_{F, t} \mid I_{t-1}\right]$ son las varianzas de los rendimientos de los portafolios de cobertura y sin cobertura. Los valores altos del ICE indican una cobertura superior, que al mismo tiempo se traduce en una reducción mayor del riesgo.

\section{Modelos de correlación condicional dinámicos}

En la medida en que llega a los mercados nueva información, el conjunto de ella se actualiza y las razones de cobertura óptima cambian con el tiempo. En términos de la reducción del riesgo, esta clase de estrategias de cobertura son superiores a las estáticas. De esta manera, en la estimación de las varianzas y covarianzas de los rendimientos de las posiciones de contado y futuros sobre petróleo se emplean dos modelos de correlación condicional dinámicos desarrollados por Engle (2002) y Tse y Tsui (2002). De acuerdo con la distribución establecida, los modelos de CCD incorporan efectos informacionales y tienen la flexibilidad de cambiar el comportamiento de las innovaciones en las ecuaciones de las varianzas, covarianzas y correlaciones condicionales. Esto, debido al hecho de que 
cada varianza condicional sigue un proceso GARCH univariado. Además, un modelo autorregresivo y de promedios móviles se agrega a la matriz de correlaciones condicionales para garantizar la condición de positiva definida en cada intervalo de tiempo durante la estimación.

El presente estudio amplía los modelos MGARCH al incorporar el término de corrección de error en las ecuaciones de las medias condicionales. Esta variable captura la tendencia estocástica común o relación de equilibrio de largo plazo entre los mercados de contado y futuros. De esta manera, el término de corrección de error tendrá la capacidad predictiva para explicar mejor la naturaleza de los precios de las posiciones de contado y de futuros a través del uso de la metodología de Engle y Granger (1987).

Los modelos MGARCH-CCD con término de corrección de error para los mercados de contado y de futuros se definen como

$$
\begin{aligned}
R_{S, t} & =\phi_{S, 0}+\phi_{S, 1}\left(S_{t-1}-\lambda_{0}-\lambda_{1} F_{t-1}\right)+\varepsilon_{S, t} \\
R_{F, t} & =\phi_{F, 0}+\phi_{F, 1}\left(S_{t-1}-\lambda_{0}-\lambda_{1} F_{t-1}\right)+\varepsilon_{F, t} \\
H_{t} & =D_{t} \Gamma_{t} D_{t} \\
\Gamma_{t} & =\left(\operatorname{diag}\left(Q_{t}\right)\right)^{-1 / 2} Q_{t}\left(\operatorname{diag}\left(Q_{t}\right)\right)^{-1 / 2} \\
D_{t} & =\operatorname{diag}\left(\sqrt{h_{11, t}}, \sqrt{h_{22, t}}, \sqrt{h_{33, t}}, \sqrt{h_{44, t}}\right),
\end{aligned}
$$

donde $R_{S, t}$ y $R_{S, t}$ son los cambios logarítmicos en los precios de las posiciones de contado y de futuros entre $t-1$ y $t . S_{t-1}$ y $F_{t-1}$ son los logaritmos de los precios del petróleo para entrega inmediata y futura. $S_{t-1}-\lambda_{0}-\lambda_{1} F_{t-1}$ es el término de corrección de error. $\Gamma_{t}$ representa la matriz de correlaciones dinámicas simétricas de dimensión $4 \times 4$. $D_{t}$ es la matriz diagonal de las desviaciones estándar condicionales derivada de la estimación de los modelos GARCH estándar.

La expresión para las CCD está definida de la siguiente manera:

$$
\begin{aligned}
& Q_{t}=(1-\alpha-\beta) \bar{Q}+\alpha \xi_{i, t-1} \xi_{j, t-1}+\beta Q_{t} \\
& \Gamma_{t}=Q_{t}^{*-1} Q_{t} Q_{t}^{*-1},
\end{aligned}
$$

donde $Q_{t}=\left(q_{i j, t}\right)$ es un matriz positiva definida simétrica de $4 \times 4$ y $\bar{Q}$ es la matriz de correlaciones incondicionales de los residuales estandarizados, $\xi_{i, t} \xi_{j, t}$. Los parámetros $\alpha$ y $\beta$ son positivos y $\alpha+\beta<1$ para garantizar que $Q_{t}$ sea positiva, y también satisfacer la condición de reversión a la media. 
Por lo tanto, las CCD se pueden expresar de la siguiente manera:

$$
\rho_{i j, t}=\frac{(1-\alpha-\beta) \bar{q}_{i j}+\alpha \xi_{i, t-1} \xi_{j, t-1}+\beta q_{i j, t-1}}{\left[(1-\alpha-\beta) \bar{q}_{i i}+\alpha \xi_{i, t-1}^{2}+\beta q_{i i, t-1}\right]^{1 / 2}\left[(1-\alpha-\beta) \bar{q}_{j j}+\alpha \xi_{j, t-1}^{2}+\beta q_{j j, t}\right]^{1 / 2}},
$$

donde $q_{i j}$ es el elemento de la $i$-ésima fila y jésima columna de la matriz $Q_{t}$.

Por su parte, el modelo de CCD de Tse y Tsui (2002) incluye las mismas especificaciones para las medias y varianzas condicionales, pero con diferente estructura en la ecuación de la correlación condicional. La matriz de correlación condicional dinámica se calcula en forma recursiva de la siguiente manera:

$$
\Gamma_{t}=(1-\alpha-\beta) \Gamma+\alpha \Psi_{t-1}+\beta \Gamma_{t-1},
$$

donde $\alpha$ y $\beta$ son parámetros positivos y $\alpha+\beta<1$, lo que garantiza que $h_{i i, t}>0 . \Gamma$ es la matriz simétrica positiva definida de parámetros constantes de $K \times K$ con $\rho_{i i}=1$ para toda $i . \Gamma_{t}$ constituye el promedio ponderado de $\Gamma, \Gamma_{t-1}$ y $\Psi_{t-1}$, la cual define a la matriz de correlaciones de $K \times K$ que depende de los residuales estandarizados, $\xi_{\tau}$ para $\tau=t-M, t-M+1, \ldots, t-1$.

En consecuencia, las CCD están determinadas por la siguiente expresión:

$$
\Psi_{i j, t-1}=\frac{\sum_{m=1}^{M} \xi_{i, t-m} \xi_{j, t-m}}{\left[\left(\sum_{m=1}^{M} \xi_{i, t-m}^{2}\right)\left(\sum_{m=1}^{M} \xi_{j, t-m}^{2}\right)\right]^{1 / 2}} \text { para } i, j=S, F \mathrm{y} i \neq j,
$$

donde $\xi_{i, t}=\varepsilon_{i, t} / \sqrt{h_{i, t}}$. La condición $M \geq K$ es necesaria para garantizar que las matrices $\Psi_{t-1}$ y $\Gamma_{t}$ cumplan con la característica de positiva definida.

Finalmente, el proceso de estimación de los parámetros y correlaciones condicionales en ambos modelos MGARCH-CCD se lleva cabo mediante el método de cuasi máxima verosimilitud. Con el supuesto de innovaciones normales, las funciones logarítmicas de verosimilitud conjunta para el periodo de la muestra se pueden expresar de la siguiente manera:

$$
\begin{gathered}
L(\theta)=-\frac{1}{2} \sum_{t=1}^{T}\left[\left(5 \log (2 \pi)+\log \left|D_{t}\right|^{2}+\varepsilon_{t}^{\prime} D_{t}^{-1} \varepsilon_{t}\right)+\left(\log \left|R_{t}\right|+\xi_{t}^{\prime} R_{t}^{-1} \xi_{t}-\xi_{t}^{\prime} \xi_{t}\right)\right] \\
L(\theta)=-\frac{1}{2} \sum_{t=1}^{T}\left[\log \left|D_{t} \Gamma_{t} D_{t}\right|+\xi_{t}^{\prime} \Gamma_{t}^{-1} \xi_{t}\right]=-\frac{1}{2} \sum_{t=1}^{T}\left[\log \left|D_{t} \Gamma_{t} D_{t}\right|+\varepsilon_{t}^{\prime} D_{t}^{-1} \Gamma_{t}^{-1} D_{t}^{-1} \varepsilon_{t}\right]
\end{gathered}
$$


donde $T$ es el número de observaciones y $\theta$ representa el vector de parámetros a estimar.

\section{DATOS Y EVIDENCIA EMPÍRICA}

\section{Descripción de los datos y análisis preliminar}

Debido a la importancia del petróleo para la economía nacional, este estudio se centra en el diseño de coberturas cruzadas. Para ello, se hace uso de contratos de futuros sobre petróleo dulce ligero listados en los mercados de futuros de Nueva York y Londres como mecanismo para transferir la exposición al riesgo de precios fluctuantes en el mercado del petróleo mexicano. Los datos empleados en el estudio incluyen los precios diarios físicos de los petróleos Maya, Istmo y Olmeca y los precios diarios de cierre de los futuros del wTI y del Brent. En la construcción de las series de los precios de futuros se adoptó la regla, habitual en la literatura, de utilizar el contrato de futuros con plazo más corto. Asimismo, para evitar los efectos de precios volátiles derivados de la expiración del contrato y la falta de liquidez en el mercado, ${ }^{7}$ el proceso de transición al segundo contrato de futuros con vencimiento más próximo se lleva a cabo una semana antes del último día de operación del contrato de futuros actual. El análisis cubre el periodo del 3 de enero de 2000 al 31 de diciembre de 2013, para un total de 3605 observaciones. Todas las series financieras se obtuvieron de la base de datos de Bloomberg.

La grafica 1 describe el comportamiento dinámico de los precios físicos de los petróleos Maya, Istmo y Olmeca, y los precios de cierre de los futuros de WTI y Brent en el periodo 2000-2013. En el análisis de la gráfica se observa que los precios en los mercados de contado y de futuros se mueven en la misma dirección y, al mismo tiempo, se muestra una tendencia estocástica común durante el periodo de estudio. Excluyendo la caída de 2001 y el desplome del 15 de julio de 2008, los precios del hidrocarburo han mantenido una prolongada tendencia alcista y episodios de alta volatilidad, particularmente después de la crisis subprime.

\footnotetext{
${ }^{7}$ En la medida en que el contrato de futuros se acerca a su vencimiento, la concentración de la actividad del mercado se mueve inmediatamente al segundo contrato de futuros con vencimiento más cercano. Esto hace que se incremente automáticamente el volumen de operación y el interés abierto.
} 
Gráfica 1. Comportamiento de los precios físicos y de futuros del petróleo
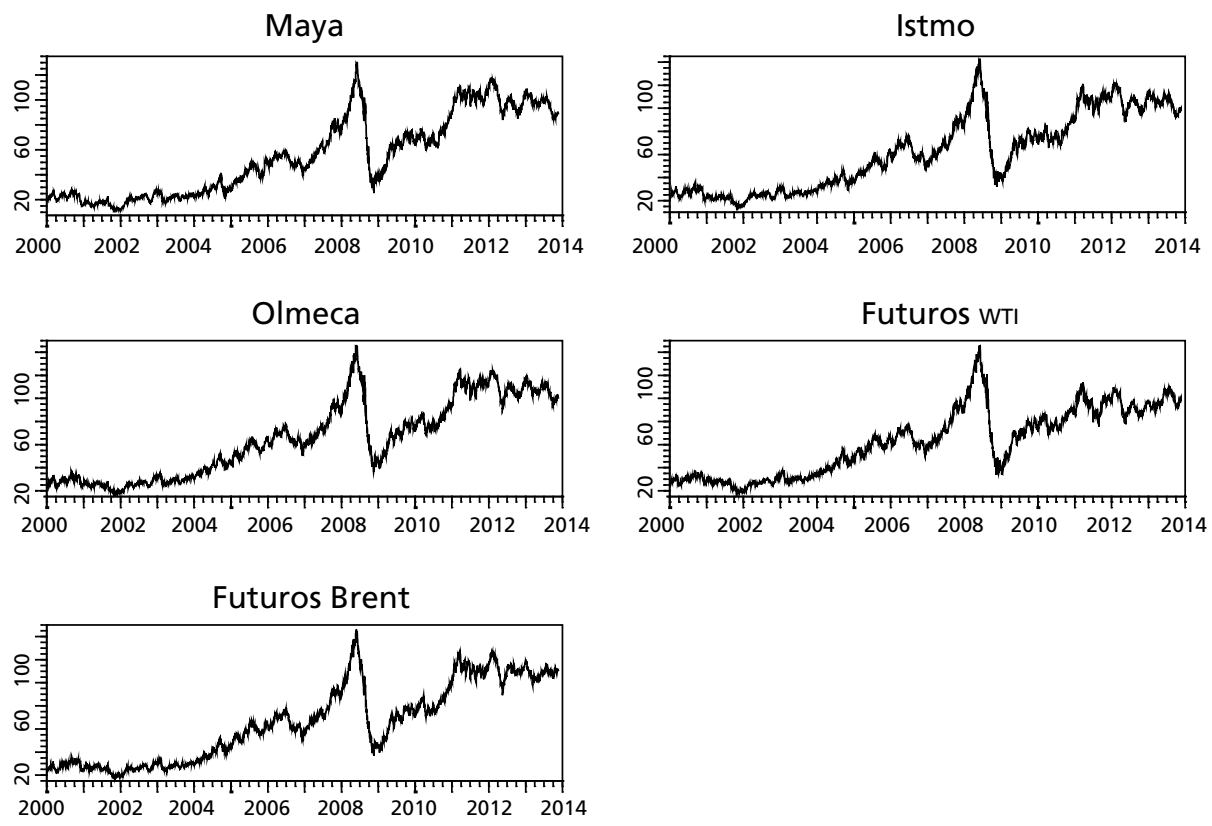

Fuente: Elaboración propia con información de la base de datos de Bloomberg.

Para el análisis de las estrategias de cobertura y su eficiencia, las series de precios son transformadas en series estacionarias a través de la diferencia de los logaritmos del precio actual y el precio previo, es decir, $R_{t}=\ln \left(P_{t}\right)-\ln \left(P_{t-1}\right)$. El tamaño de la muestra permite el análisis fuera de la muestra, extrayendo las últimas 778 observaciones de la muestra total. El cuadro 1 muestra las estadísticas básicas y las pruebas de raíz unitaria de las series de precios y rendimientos del petróleo. Los resultados muestran rendimientos promedio relativamente más pequeños que las desviaciones estándar, lo que indica un riesgo relativamente más alto, particularmente para los petróleos Maya e Istmo. Los rendimientos en ambos mercados presentan las características típicas de asimetría negativa y colas anchas como consecuencia del exceso de curtosis. Además, el supuesto de normalidad de la distribución incondicional es rechazado por el estadístico Jarque-Bera para los cinco tipos de petróleo.

La gráfica 2, que muestra el comportamiento de los rendimientos en los mercados físicos y de futuros del petróleo a lo largo del tiempo, evidencia la presencia de fuerte volatilidad en aglomeraciones, esto es, periodos de alta volatili- 
dad seguidos de periodos de relativa tranquilidad. Cabe resaltar que la intensidad del fenómeno de la heterocedasticidad condicional es más pronunciada en los periodos de 2001, 2008-2009 y 2010-2012, durante los cuales todas las series de rendimientos experimentaron una sucesión de movimientos atípicos positivos y negativos en cortos intervalos de tiempo. Además, la presencia de fuerte heterocedasticidad o efectos ARCH en la volatilidad de los rendimientos del petróleo es sustentada por el valor alto y significativo del estadístico de la prueba del multiplicador de Lagrange (LM) a un nivel de 5\% con veinte rezagos. Estos importantes hallazgos sugieren la aplicación de modelos GARCH univariados o multivariados para su apropiada modelación.

Cuadro 1. Estadísticas básicas y pruebas de raíces unitarias

\begin{tabular}{|c|c|c|c|c|c|}
\hline & Maya & Istmo & Olmeca & WTI & Brent \\
\hline \multicolumn{6}{|c|}{ Panel A: Estadísticos básicos } \\
\hline Media & 0.0418 & 0.0388 & 0.0385 & 0.0364 & 0.0410 \\
\hline Mínimo & -22.2611 & -22.4634 & -21.2122 & -16.5405 & -14.4434 \\
\hline Máximo & 18.2032 & 20.3453 & 20.5608 & 16.4123 & 12.7145 \\
\hline Desviación & 2.5961 & 2.5501 & 2.3664 & 2.3797 & 2.1932 \\
\hline Sesgo & -0.2764 & -0.3618 & -0.2054 & -0.2052 & -0.2557 \\
\hline Curtosis & 11.1312 & 10.7711 & 9.6353 & 7.6036 & 6.1484 \\
\hline Jarque-Bera & 9981 & 9146 & 6636 & 3207 & 1527 \\
\hline LM & 542 & 458 & 478 & 541 & 512 \\
\hline \multicolumn{6}{|c|}{ Panel B: Pruebas de raíz unitaria } \\
\hline \multicolumn{6}{|l|}{ Niveles } \\
\hline Dickey-Fuller & -2.85 & -2.72 & -2.63 & -2.65 & -2.45 \\
\hline Phillips-Perron & -2.80 & -2.65 & -2.55 & -2.54 & -2.41 \\
\hline \multicolumn{6}{|c|}{ Primeras diferencias } \\
\hline Dickey-Fuller & $-27.41 *$ & $-27.79 *$ & $-27.32 *$ & $-27.98^{*}$ & $-27.62^{*}$ \\
\hline Phillips-Perron & $-63.44 *$ & $-63.59 *$ & $-64.68^{*}$ & $-61.66^{*}$ & $-63.52 *$ \\
\hline
\end{tabular}

Nota: * significación a un nivel de $1 \%$.

Fuente: Elaboración propia con información de la base de datos de Bloomberg.

En el panel в del cuadro 1 se muestran los resultados de las pruebas de raíz unitaria de Dickey-Fuller y Phillips-Perron para el análisis de la estacionariedad en las series de los mercados de contado y de futuros del petróleo. En ambas, los estadísticos muestran valores no significativos estadísticamente para los logaritmos de los precios de contado y de futuros, pero significativos a un nivel de $1 \%$ para las primeras diferencias en todas las series. La existencia de raíz unitaria en los niveles de los precios de contado y de futuros significa que las 
Gráfica 2. Dinámica de los rendimientos de los mercados físicos y de futuros del petróleo
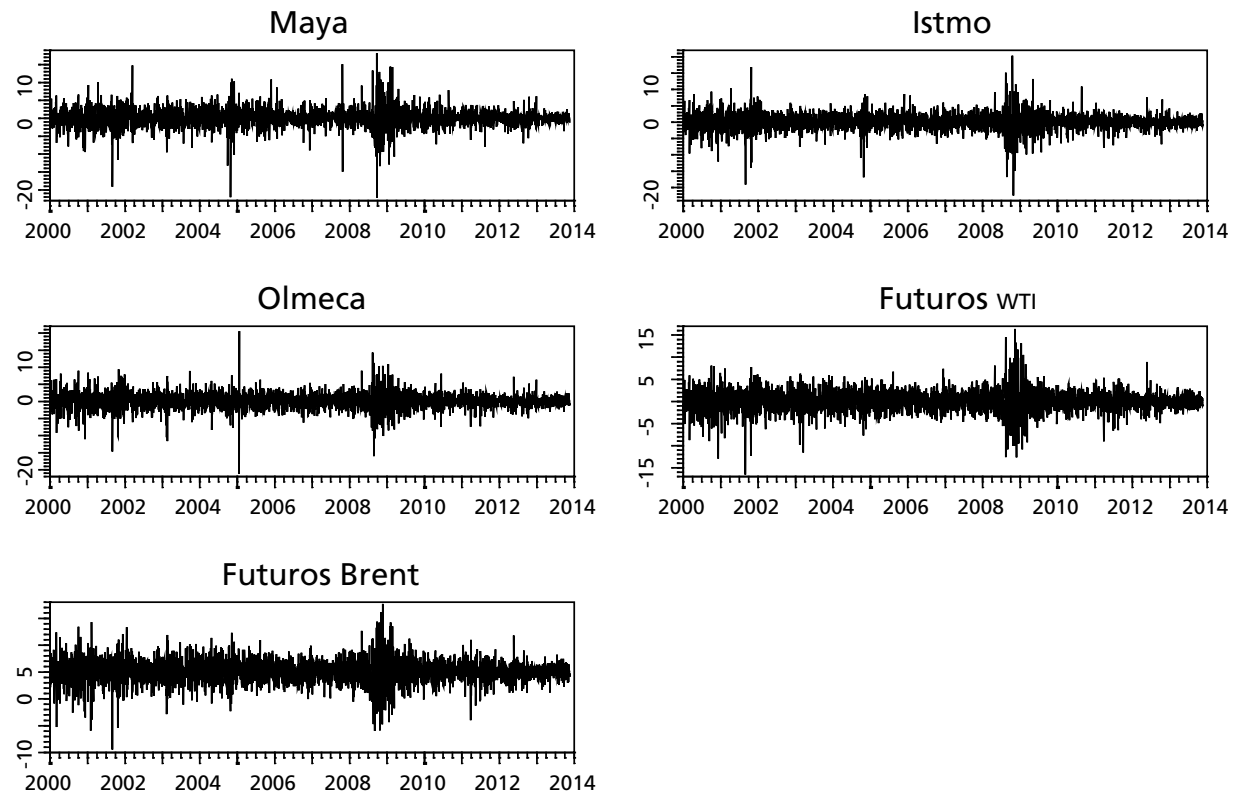

Fuente: Elaboración propia con datos de la base de datos de Bloomberg.

series no son estacionarias y presentan un comportamiento de paseo aleatorio. Este hallazgo confirma probablemente la existencia de una relación estacionaria lineal entre dos series no estacionarias, es decir, entre los precios de contado y los de futuros.

Para investigar la existencia de relación de cointegración de largo plazo, se aplicó la metodología de Engle y Granger en dos pasos. ${ }^{8}$ En el cuadro 2 se muestran los resultados de la prueba de cointegración entre el logaritmo de los precios físicos y el logaritmo de los precios de futuros. Los parámetros estimados de la prueba de cointegración son estadísticamente significativos e incluso aproximadamente iguales a la unidad para $\lambda_{1}$ con errores estándar pequeños. La cointegración para cada par de series es confirmada por el valor de los estadísticos de las pruebas de Dickey- Fuller y Phillips-Perron a un nivel de significación de $1 \%$. Esto, debido al hecho de que las series de los residuales son estacionarias

\footnotetext{
${ }^{8}$ Para una descripción técnica más detallada del método de cointegración, véase Engle y Granger (1987).
} 
Cuadro 2. Pruebas de cointegración

\begin{tabular}{lcccc}
\hline & $\lambda_{0}$ & $\lambda_{1}$ & Dickey-Fuller & Phillips-Perron \\
\hline Maya-WTI & $0.5728(0.0920)$ & $0.8323(0.0029)$ & $-4.3654^{*}$ & $-6.1392^{*}$ \\
Istmo-WTI & $0.4209(0.0081)$ & $0.9142(0.0021)$ & $-6.8565^{*}$ & $-12.9365^{*}$ \\
Olmeca- WTI & $0.2216(0.0054)$ & $0.9493(0.0014)$ & $-8.6182^{*}$ & $-18.6275^{*}$ \\
Maya-Brent & $0.6931(0.0102)$ & $0.8740(0.0028)$ & $-4.4342^{*}$ & $-6.4148^{*}$ \\
Istmo-Brent & $0.2240(0.0081)$ & $0.9586(0.0021)$ & $-6.5687^{*}$ & $-13.0484^{*}$ \\
Olmeca-Brent & $0.0169(0.0054)$ & $0.9948(0.0014)$ & $-8.5164^{*}$ & $-18.5676^{*}$ \\
\hline
\end{tabular}

Nota: * significación a un nivel de 1\%. Los errores estándar figuran entre paréntesis.

Fuente: Elaboración propia con información de la base de datos de Bloomberg.

o integradas de orden cero, I(0). Estos hallazgos sugieren claramente la necesidad de incorporar el término de corrección de error en las ecuaciones de las medias condicionales de los modelos MGARCH-CCD.

\section{Estimación de los modelos MGARCH-CCD}

En los cuadros 3 y 4 se proporcionan los resultados estimados de los modelos MGARCH-CCD con término de corrección de error discutidos en la sección previa. En ambos modelos, los coeficientes constantes de las dos ecuaciones de la media condicional son positivos y estadísticamente significativos a un nivel de $1 \%$, con la excepción de las relaciones Olmeca contra WTI y Brent para los rendimientos de los precios físicos. En los tres mercados del petróleo nacional y los dos modelos, los parámetros estimados $\left(\phi_{S, 1}\right)$ asociados con el término de corrección de error son positivos y estadísticamente significativos a un nivel de $1 \%$.

En contraste, las desviaciones de corto plazo en la relación de equilibrio tienen un efecto negativo en la mayoría de las ecuaciones de las medias condicionales de los rendimientos de los futuros. La significación $\left(\phi_{F, 1}\right)$ sólo es alcanzada en las relaciones Istmo-WTI, Olmeca-WTI y Olmeca-Brent por el modelo MGARCH-CCD de Tse y Tsui, por lo que un incremento en las desviaciones de corto plazo tiende a reducir los cambios en los precios de los futuros, pero que al mismo tiempo incrementa los rendimientos de los precios de contado.

En el caso de las ecuaciones de la varianza condicional, todos los parámetros estimados son estadísticamente significativos a un nivel de $1 \%$ en ambos mercados de petróleo. Los valores de los coeficientes GARCH son positivos y oscilan entre 0.8333 y 0.9169 (contado) y entre 0.8789 y 0.9401 (futuros), mien- 
132 ECONOMÍA: TEORÍA Y PRÁCTICA • Nueva Época, número 44, enero-junio 2016

\section{Cuadro 3. Estimaciones de los parámetros} del modelo CCD de Engle

\begin{tabular}{|c|c|c|c|c|c|c|}
\hline \multirow[b]{2}{*}{ Parametros } & \multicolumn{3}{|c|}{ WTI } & \multicolumn{3}{|c|}{ Brent } \\
\hline & Maya & Istmo & Olmeca & Maya & Istmo & Olmeca \\
\hline \multirow[t]{2}{*}{$\phi_{S, 0}$} & $0.1728 *$ & $0.0724 * *$ & 0.0439 & $0.1778^{*}$ & $0.1322 *$ & 0.0590 \\
\hline & $(0.0391)$ & $(0.0382)$ & $(0.0389)$ & $(0.0385)$ & $(0.0391)$ & $(0.0396)$ \\
\hline \multirow{2}{*}{$\phi_{S, 1}$} & $1.3347^{*}$ & $2.3171^{*}$ & $3.5465^{*}$ & $2.8696^{*}$ & $1.7590^{*}$ & $2.3393 *$ \\
\hline & (05109) & $(0.5201)$ & $(0.3563)$ & $(0.5058)$ & $(0.8202)$ & $(0.7568)$ \\
\hline \multirow[t]{2}{*}{$\phi_{F, 0}$} & $0.1000 *$ & $0.1400 *$ & $0.1295^{*}$ & $0.1240 *$ & $0.1850 *$ & $0.1538^{*}$ \\
\hline & $(0.0372)$ & $(0.0361)$ & $(0.0389)$ & $(0.0348)$ & $(0.0344)$ & $(0.0367)$ \\
\hline \multirow[t]{2}{*}{$\phi_{F, 1}$} & -0.0054 & -1.1049 & -1.2317 & 0.1301 & -0.5333 & -1.3714 \\
\hline & $(0.4637)$ & $(0.7362)$ & $(0.6789)$ & $(0.4312)$ & $(0.6824)$ & $(0.6540)$ \\
\hline \multirow[t]{2}{*}{$\omega_{s}$} & $0.2386^{*}$ & $0.1336^{*}$ & $0.2524^{*}$ & $0.2716^{*}$ & $0.1860 *$ & $0.3282^{*}$ \\
\hline & $(0.0479)$ & $(0.0339)$ & $(0.0523)$ & $(0.0589)$ & $(0.0422)$ & $(0.0615)$ \\
\hline \multirow[t]{2}{*}{$\alpha_{s}$} & $0.1232^{*}$ & $0.0825^{*}$ & $0.1046^{*}$ & $0.1455^{*}$ & $0.0986^{*}$ & $0.1142 *$ \\
\hline & $(0.0141)$ & $(0.0108)$ & $(0.0138)$ & $(0.0175)$ & $(0.0124)$ & $(0.1384)$ \\
\hline \multirow[t]{2}{*}{$\beta_{S}$} & $0.8526^{*}$ & $0.9019 *$ & $0.8624^{*}$ & $0.8333^{*}$ & $0.8793^{*}$ & 0.8401 * \\
\hline & $(0.0162)$ & $(0.0134)$ & $(0.0186)$ & $(0.2008)$ & $(0.1527)$ & (0.0193) \\
\hline$\alpha_{S}+\beta_{S}$ & 0.9758 & 0.9844 & 0.9670 & 0.9788 & 0.9779 & 0.9543 \\
\hline \multirow[t]{2}{*}{$\omega_{F}$} & $0.1174^{*}$ & $0.0665^{*}$ & $0.1564^{*}$ & $0.1076^{*}$ & $0.0904 *$ & $0.1606^{*}$ \\
\hline & $(0.0318)$ & $(0.0243)$ & $(0.0388)$ & $(0.0282)$ & $(0.0271)$ & $(0.0381)$ \\
\hline \multirow[t]{2}{*}{$\alpha_{F}$} & $0.0687 *$ & $0.0632 *$ & $0.0868^{*}$ & $0.0713^{*}$ & $0.0790 *$ & 0.0980 * \\
\hline & $(0.0090)$ & $(0.0092)$ & $(0.0113)$ & $(0.0098)$ & $(0.0104)$ & $(0.0120)$ \\
\hline \multirow[t]{2}{*}{$\beta_{F}$} & $0.9128 *$ & $0.9074 *$ & $0.8939 *$ & $0.9092 *$ & $0.9074^{\star}$ & 0.8789 * \\
\hline & $(0.0119)$ & $(0.0130)$ & $(0.0144)$ & $(0.0130)$ & $(0.0130)$ & $(0.0155)$ \\
\hline$\alpha_{F}+\beta_{F}$ & 0.9715 & 0.9706 & 0.9807 & 0.9805 & 0.9864 & 0.9769 \\
\hline \multirow[t]{2}{*}{$Q(12)$} & 8.1645 & 9.5742 & 12.7912 & 10.2715 & 11.8726 & 12.3526 \\
\hline & [0.7725] & 0.6533 & [0.3846] & [0.5926] & {$[0.4562]$} & [0.4182] \\
\hline \multirow[t]{2}{*}{$Q^{2}(12)$} & 14.5778 & 7.4091 & 10.8522 & 13.332 & 12.9622 & 16.9622 \\
\hline & [02660] & {$[0.8295]$} & [0.5417] & {$[0.3456]$} & {$[0.3721]$} & {$[0.2616]$} \\
\hline
\end{tabular}

Nota: $Q(12)$ y $Q 2(12)$ indican los estadísticos de la prueba de Ljung-Box para los residuos simples y cuadrados con 12 rezagos y valores de $p$ entre corchetes. * $\mathrm{y}$ ** indican significación para los niveles de $1 \%$ y $5 \%$, respectivamente. Los errores estándar se muestran entre paréntesis.

Fuente: Elaboración propia con información de la base de datos de Bloomberg. 


\section{Cuadro 4. Estimaciones de los parámetros del modelo CCD de Tse y Tsui}

\begin{tabular}{|c|c|c|c|c|c|c|}
\hline \multirow[b]{2}{*}{ Parámetros } & \multicolumn{3}{|c|}{ WTI } & \multicolumn{3}{|c|}{ Brent } \\
\hline & Maya & Istmo & Olmeca & Maya & Istmo & Olmeca \\
\hline \multirow[t]{2}{*}{$\phi_{S, 0}$} & $0.1647^{*}$ & $0.0919 *$ & 0.0347 & $0.1685^{*}$ & $0.1246 *$ & 0.0491 \\
\hline & $(0.0390)$ & $(0.0390)$ & $(0.0391)$ & $(0.0388)$ & $(0.0397)$ & $(0.0396)$ \\
\hline \multirow[t]{2}{*}{$\phi_{S, 1}$} & $2.3549 *$ & $2.3687^{*}$ & $1.7003^{*}$ & $2.9686^{*}$ & $1.7638^{*}$ & $3.0543 *$ \\
\hline & $(0.5082)$ & $(0.8267)$ & $(0.3040)$ & $(0.5156)$ & $(0.8059)$ & $(0.7092)$ \\
\hline \multirow[t]{2}{*}{$\phi_{F, 0}$} & $0.1104 *$ & $0.0956 *$ & $0.1044 *$ & $0.1287^{*}$ & $0.1548^{*}$ & $0.1173^{*}$ \\
\hline & $(0.0370)$ & $(0.0359)$ & $(0.0381)$ & $(0.0348)$ & $(0.0343)$ & $(0.0362)$ \\
\hline \multirow[t]{2}{*}{$\phi_{F, 1}$} & -0.1035 & $-1.4852 * *$ & $-2.6156^{*}$ & 0.0108 & -0.5696 & $-2.5640 *$ \\
\hline & $(0.4621)$ & $(0.7195)$ & $(0.8530)$ & $(0.4342)$ & $(0.6531)$ & $(0.9086)$ \\
\hline \multirow[t]{2}{*}{$\omega_{S}$} & $0.2016^{*}$ & $0.1220 *$ & $0.1359 *$ & $0.2461 *$ & $0.1608^{*}$ & 0.2790 * \\
\hline & $(0.0405)$ & $(0.0333)$ & $(0.0488)$ & $(0.0528)$ & $(0.0404)$ & $(0.0587)$ \\
\hline \multirow[t]{2}{*}{$\alpha_{s}$} & $0.1048^{*}$ & $0.0704 *$ & 0.0610 * & $0.1293^{*}$ & 0.0850 & 0.0928 * \\
\hline & $(0.0120)$ & $(0.0107)$ & $(0.0127)$ & $(0.0157)$ & $(0.0123)$ & $(0.0135)$ \\
\hline \multirow[t]{2}{*}{$\beta_{S}$} & 0.8711 * & $0.9129 *$ & $0.9169 *$ & 0.8491 * & $0.8928^{*}$ & $0.8613^{*}$ \\
\hline & $(0.0142)$ & $(0.0138)$ & $(0.0195)$ & $(0.0187)$ & $(0.0158)$ & $(0.0203)$ \\
\hline$\alpha_{S}+\beta_{S}$ & 0.9759 & 0.9833 & 0.9779 & 0.9784 & 0.9778 & 0.9541 \\
\hline \multirow[t]{2}{*}{$\omega_{F}$} & $0.1032 *$ & $0.0544 *$ & 0.0787 * & $0.1112^{*}$ & $0.0714^{*}$ & $0.1098^{*}$ \\
\hline & $(0.0268)$ & $(0.0198)$ & $(0.0243)$ & $(0.0283)$ & $(0.0228)$ & $(0.0279)$ \\
\hline \multirow[t]{2}{*}{$\alpha_{F}$} & $0.0596^{*}$ & $0.0518^{*}$ & $0.0535^{*}$ & 0.0656 * & $0.0674^{*}$ & 0.0669 * \\
\hline & $(0.0082)$ & $(0.0077)$ & $(0.0078)$ & $(0.0094)$ & $(0.0096)$ & $(0.0092)$ \\
\hline \multirow[t]{2}{*}{$\beta_{F}$} & 0.9231 * & 0.9401 * & 0.9336 * & $0.9132 *$ & $0.9207 *$ & $0.9118^{*}$ \\
\hline & $(0.0111)$ & $(0.0094)$ & $(0.0104)$ & $(0.0130)$ & $(0.0119)$ & $(0.0127)$ \\
\hline$\alpha_{F}+\beta_{F}$ & 0.9827 & 0.9919 & 0.9871 & 0.9788 & 0.9881 & 0.9787 \\
\hline \multirow[t]{2}{*}{$Q(12)$} & 8.5995 & 7.2835 & 8.2854 & 8.1092 & 10.8152 & 13.3925 \\
\hline & [0.5367] & [0.8338] & [0.7626] & [0.7766] & {$[0.1709]$} & [0.3411] \\
\hline \multirow[t]{2}{*}{$Q^{2}(12)$} & 16.0545 & 15.7871 & 12.5697 & 14.3546 & 19.2975 & 14.9622 \\
\hline & [0.5514] & {$[0.2016]$} & {$[0.4021]$} & [0.2791] & {$[0.0818]$} & [0.1512] \\
\hline
\end{tabular}

Nota: $Q(12)$ y $Q^{2}(12)$ indican los estadísticos de la prueba de Ljung-Box para los residuos simples y cuadrados con 12 rezagos y valores de $p$ entre corchetes. * $y^{* *}$ indican significación para los niveles de $1 \%$ y $5 \%$, respectivamente. Los errores estándar se muestran entre paréntesis.

Fuente: Elaboración propia con información de la base de datos de Bloomberg. 
tras que los coeficientes ARCH varían de 0.0610 a 0.1455 (contado) y de 0.0518 a 0.0980 (futuros). Estos hallazgos confirman que las varianzas-covarianzas condicionales y las razones de cobertura óptima presentan un comportamiento cambiante en el tiempo y un alto grado de persistencia debido a que la suma de los términos ARCH y GARCH es menor a uno.

Finalmente, la especificación apropiada de los primeros dos momentos condicionales es validada estadísticamente mediante la correlación serial de ruido blanco. En este sentido, los valores de los estadísticos de la prueba de LjungBox indican que no existe correlación serial en los residuos estandarizados simples y cuadrados con 12 rezagos entre los rendimientos de los precios de contado y los de futuros, los cuales también son apoyados por los valores de $p$ que son mayores al nivel de significación de 5\%.

\section{Estimación de la razón de cobertura cruzada óptima}

En esta sección se estiman las razones de cobertura cruzada óptima (RCCO) utilizando las varianzas y covarianzas condicionales estimadas de los dos modelos MGARCH-CCD. En la estimación de las RCCO fuera de la muestra se emplea el periodo del 3 de enero de 2011 al 31 de diciembre de 2013. En este caso, los parámetros de los modelos MGARCH-CCD son reestimados utilizando una ventana móvil de 2827 observaciones. Este procedimiento consiste en remover la observación más remota y agregar la observación más reciente al periodo de la muestra. Así, el tamaño de ésta se mantiene fijo durante la reestimación de los modelos y las predicciones fuera de la muestra no se traslapan, lo que genera 12 series de tamaño de 778 RCCO estimadas. La ventana móvil diaria es seleccionada en la predicción de las RCCO, porque la estructura volátil y riesgosa del mercado del petróleo requiere del rebalanceo continuo del portafolio de cobertura. Además, la flexibilidad del mercado de futuros permite llevar a cabo esta clase de estrategias, puesto que los participantes pueden cancelar sus posiciones financieras cortas o largas con sólo tomar la posición opuesta.

El comportamiento dinámico de las RCCO estimadas se ilustra en las graficas 3 y 4 . En cada ilustración se comparan las estimaciones fuera de la muestra de las RCCO obtenidas por los modelos MGARCH-CCD y el modelo de reversión. Analizando ambas figuras se puede observar que los modelos MGARCH-CCD con corrección de error proporcionan las RCCO más grandes. Además, éstas son más volátiles que las del modelo de reversión en la medida en que las varianzas y covarianzas condicionales cambian con el tiempo en el corto plazo. De hecho, la variación en 
Gráfica 3. Razones de cobertura óptima para los petróleos nacionales-WTI, 2011-2014
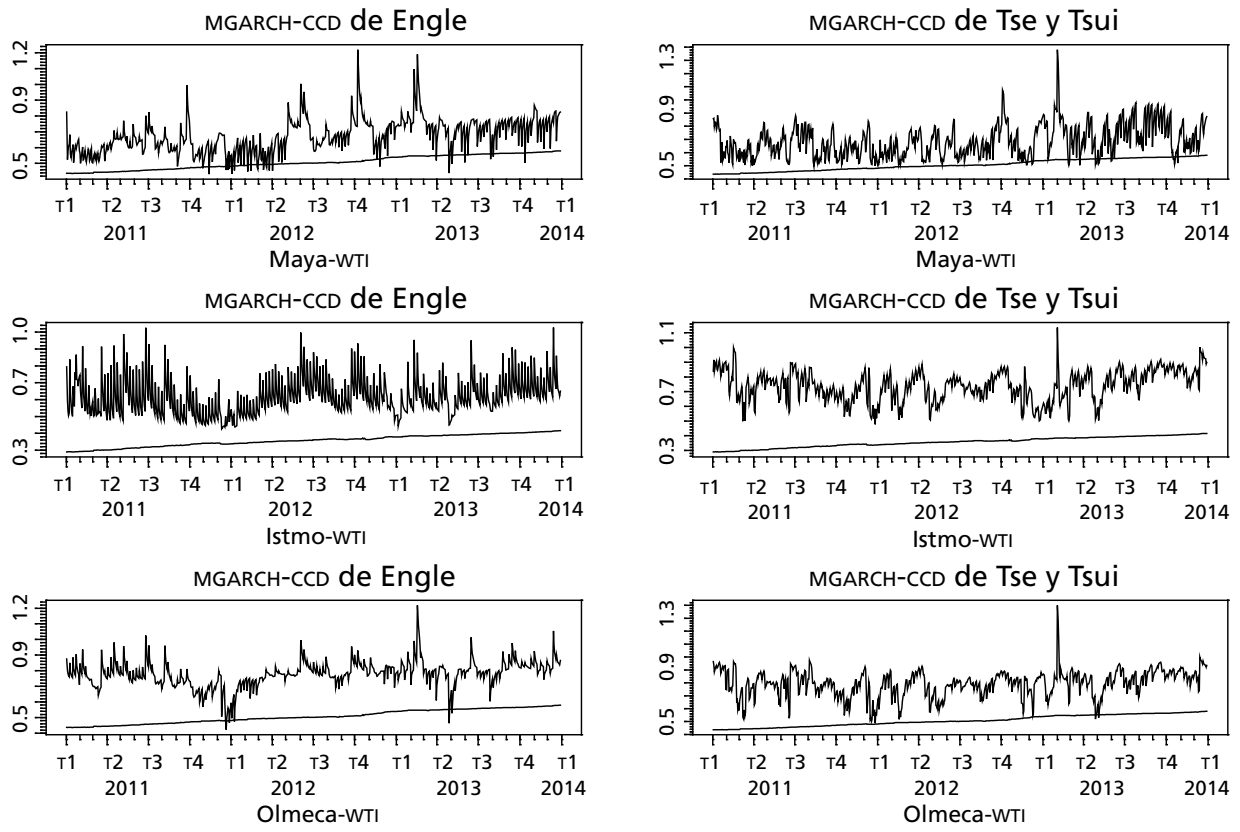

Fuente: Elaboración propia con información de la base de datos de Bloomberg.

las RCCO indica que los participantes en los mercados del petróleo nacional deben ajustar de forma continua los portafolios de cobertura para reducir la exposición al riesgo de precios fluctuantes, particularmente para las relaciones Istmo-wTI e Istmo-Brent. Estos hallazgos empíricos muestran el potencial de los modelos MGARCH-CCD en la estimación de las RCCO ante la llegada de nueva información a los mercados. Sin embargo, las especificaciones propuestas tienen algunas desventajas. La primera es que no capturan la asimetría de los impactos positivos y negativos de la misma magnitud o efecto de apalancamiento y la segunda tiene que ver con los cambios de régimen en las volatilidades y correlaciones.

Además, otra característica importante observada en los valores de las RCCO es que mantienen una tendencia positiva durante todo el periodo de análisis, aunque cabe mencionar que no es sorprendente observar que algunas son negativas para ciertos petróleos nacionales. El cambio de signo de positivo a negativo en las RCCO durante el periodo de estimación puede ser causado en gran parte por los desiguales niveles de calidad de los crudos mexicanos con respecto 
Gráfica 4. Razones de cobertura óptima para los petróleos nacionales-Brent, 2011-2014
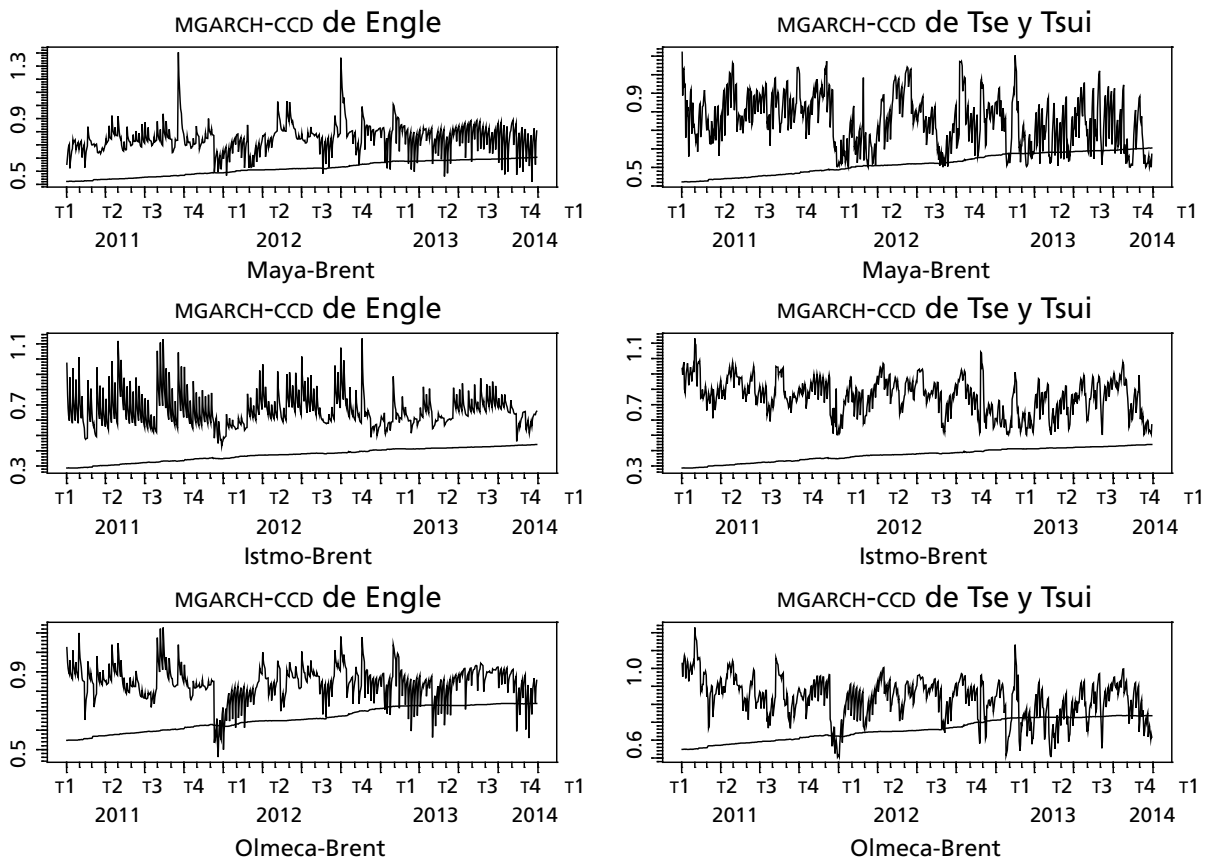

Fuente: Elaboración propia con información de la base de datos de Bloomberg.

a los marcadores WTI y Brent, excepto el Olmeca. En este sentido, la eficiencia de las estrategias de cobertura cruzada puede disminuir cuando sean petróleos con un alto contenido de azufre ${ }^{9}$ y bajo grado de densidad API (por el American Petroleum Institute), ${ }^{10}$ especialmente el Maya. Al analizar las RCCO de los modelos usados se puede apreciar que exhiben patrones similares y la propiedad de reversión a la media con respecto a las RCCO convencionales. Aunque su comportamiento es muy parecido en ambos mercados de futuros, este patrón no necesariamente quiere decir que sus valores sean idénticos en la medida en que nueva información ingresa a los respectivos mercados.

\footnotetext{
${ }^{9}$ Los petróleos con alto contenido de azufre (mayor a $0.5 \%$ ) son clasificados como ácidos, mientras que aquéllos con un contenido de azufre menor son considerados dulces.

${ }^{10}$ Los petróleos con densidad mayor a 35 grados API son considerados ligeros; entre 26 y 35 grados son medianos y con menos de 26 grados, pesados.
} 
Para un análisis más profundo del desempeño de los modelos MGARCH-CCD y la función de los mercados de futuros en la estimación de las RCCO del petróleo nacional, en el cuadro 5 se reportan los valores promedio, mínimo y máximo estimados por los modelos MGARCH-CCD, de Engle, MGARCH-CCDTT de Tse y Tsui, y de MCO, para su comparación. Los resultados de este último indican que las RCCO son claramente menores a la unidad, en particular para las relaciones Istmo-WTI e Istmo-Brent. Sus valores promedio alcanzados equivalen a 0.3569 y 0.3735 , con valores mínimos de 0.2895 y 0.2868 y valores máximos de 0.4156 y 0.4404 , respectivamente.

Cuadro 5. Comparación de las razones de cobertura cruzada óptima

\begin{tabular}{|c|c|c|c|c|c|c|}
\hline \multirow[b]{2}{*}{ Modelo } & \multicolumn{3}{|c|}{ WTI } & \multicolumn{3}{|c|}{ Brent } \\
\hline & Media & Mínimo & Máximo & Media & Mínimo & Máximo \\
\hline \multicolumn{7}{|l|}{ Maya } \\
\hline MCO & 0.5068 & 0.4364 & 0.5791 & 0.5207 & 0.4222 & 0.6057 \\
\hline MGARCH-CCD de Engle & 0.6726 & 0.4319 & 1.2198 & 0.7485 & 0.4187 & 1.4050 \\
\hline MGARCH-CCD de Tse y Tsui & 0.6866 & 0.4960 & 1.3812 & 0.7535 & 0.5011 & 1.1214 \\
\hline \multicolumn{7}{|l|}{ Istmo } \\
\hline MCO & 0.3569 & 0.2895 & 0.4156 & 0.3735 & 0.2868 & 0.4404 \\
\hline MGARCH-CCD de Engle & 0.6167 & 0.4266 & 1.0276 & 0.6655 & 0.4311 & 1.1350 \\
\hline MGARCH-CCD de Tse y Tsui & 0.7413 & 0.4775 & 1.1362 & 0.7553 & 0.5018 & 1.1311 \\
\hline \multicolumn{7}{|l|}{ Olmeca } \\
\hline MCO & 0.6187 & 0.5370 & 0.6848 & 0.6595 & 0.5479 & 0.7375 \\
\hline MGARCH-CCD de Engle & 0.7788 & 0.4217 & 1.2186 & 0.8414 & 0.4610 & 1.1276 \\
\hline MGARCH-CCD de Tse y Tsui & 0.7945 & 0.4879 & 1.4000 & 0.8315 & 0.5048 & 1.2287 \\
\hline
\end{tabular}

Fuente: Elaboración propia con información de la base de datos de Bloomberg.

Este hallazgo empírico revela que el modelo de cobertura convencional no toma en cuenta las diferencias de precios entre los petróleos nacionales y los internacionales, evidentemente originados por las variaciones en los niveles de calidad a través de los múltiples mercados. En este sentido, las estrategias de cobertura cruzada para el petróleo nacional requieren de modelos más sofisticados que capturen apropiadamente las imperfecciones en los mercados que afectan los precios y las RCCO. Por su parte, las RCCO estimadas mediante los modelos de Engle y de Tse y Tsui tienen patrones diferentes y valores más grandes en comparación a los del modelo de cobertura convencional. Considerando el mercado 
de futuros del WTI como mecanismo de cobertura, el promedio de las RCCO oscila entre 0.6167 y 0.7945 , con un valor máximo de entre 1.0276 y 1.4000 y un valor mínimo de entre 0.4217 y 0.4960 , en tanto que para el mercado de futuros del Brent, los valores, en el mismo orden, se hallan en los rangos 0.655-0.8414, $1.1214-1.4050$ y $0.4187-0.5048$. En términos del desempeño de los modelos MGARCH-CCD para el diseño de estrategias de cobertura cruzada fuera de la muestra, los resultados muestran que el modelo de Tse y Tsui proporciona el valor promedio más alto para las RCCO, con la excepción de la relación Olmeca-Brent.

Otro hallazgo importante, es que los valores de las RCCO utilizando cualquier modelo MGARCH-CCD y el contrato de futuros del Brent son mayores a las del contrato de futuros del WTI para cualquier tipo de petróleo nacional. En la práctica real, esto tiene importantes implicaciones económico-financieras, porque una posición larga abierta en el mercado del petróleo nacional requiere mayor número de contratos cuando se cubra a través del mercado de futuros del Brent, que cuando se tome la posición en el mercado de futuros del WTI. Más específicamente, los valores promedio de las RCCO estimadas por el modelo de Engle equivalen a 0.7788 y 0.8414 para las relaciones Olmeca-wTI y Olmeca-Brent, respectivamente. De esta manera, para minimizar los riesgos de la exportación mensual promedio de 99000 barriles de petróleo Olmeca, el gobierno mexicano requiere negociar aproximadamente 77 y 83 contratos de futuros de wTI y de Brent, respectivamente. Aunque la cobertura cruzada óptima no es posible en ambos mercados, como consecuencia de la estandarización de los contratos de futuros que equivale a mil barriles de petróleo, las operaciones de cobertura realizadas en mercados organizados son generalmente más transparentes y tienen costos de transacción relativamente más bajos, a diferencia de las coberturas a la medida en los mercados OTC.

Algunos factores que pueden explicar mejor los resultados obtenidos se pueden adjudicar a la conversión de tendencia entre los precios de los petróleos de referencia internacional. En 2010-2013, el Brent comenzó a cotizarse en los mercados de energía fósil con una prima adicional con respecto al WTI, a pesar de que sus características fisicoquímicas son de mejor calidad, esto es, densidad de 40 grados API contra 38 y contenido de azufre de $0.24 \%$ contra $0.37 \%$. De esta manera, la transición de precios originó diferenciales positivos entre los futuros del Brent y los crudos nacionales, pero negativos respecto a los futuros del WTI en el periodo de análisis fuera de la muestra. ${ }^{11} \mathrm{Al}$ mismo tiempo, la amplitud ne-

\footnotetext{
${ }^{11}$ Por falta de espacio no se incluyen las gráficas de los diferenciales entre los precios de futuros y de contado de los petróleos internacionales y nacionales, pero están disponibles para cualquier aclaración de los resultados.
} 
gativa de los diferenciales afectó la tendencia ascendente de las correlaciones y, por consecuencia, las RCCO, particularmente en periodos de extrema volatilidad.

\section{Análisis de los índices eficientes de cobertura}

Los índices eficientes de cobertura son analizados en esta sección utilizando las 778 RCCO para evaluar el desempeño de las coberturas cruzadas de mínima varianza cambiantes en el tiempo de los modelos MGARCH-CCD y de MCO. En el procedimiento de estimación se emplea la misma técnica de venta móvil que coadyuva a la actualización de las estimaciones de estos índices. El tamaño de la muestra fija es de 260 observaciones, del 3 de enero al 30 de diciembre de 2011. Las últimas 518 observaciones, que corresponden al lapso del 3 de enero de 2012 al 31 de diciembre de 2013, son usadas para el análisis fuera de la muestra.

En las gráficas 5 y 6 se muestra la evolución de los índices eficientes de cobertura para los modelos MGARCH-CCD, y su comparación con el modelo de MCO para los tres portafolios de cobertura. De manera similar a las RCCO, en ambas gráficas se observa que las fluctuaciones en los índices del modelo de MCO son menos sensibles para responder a cambios en los mercados y, por consecuencia, menos volátiles, a diferencia de los índices de los modelos MGARCH-CCD. Este hecho se refleja más en la relación Maya-Brent, dado que su tendencia comienza a bajar a partir del primer trimestre de 2013, a pesar de que sus RCCO mantienen un comportamiento inverso en el mismo periodo.

$\mathrm{Al}$ analizar la tendencia de los índices eficientes de cobertura dinámicos, se puede identificar diferentes características para cada portafolio de cobertura y modelo MGARCH-CCD. Las variaciones en los índices son más notables para las relaciones Maya-wTI y Maya-Brent, debido al hecho de que oscilan en un rango más amplio, entre 10.13\%-86.99\% (modelo de Engle) y 10.10\%-85.35\% (modelo de Tse y Tsui), respectivamente. Incluso existen periodos con valores menores a los del modelo de MCO, especialmente durante los últimos tres trimestres de 2013. En general, otra de las características de los índices eficientes de cobertura es que alcanzan valores más altos en los periodos de extrema volatilidad que en los periodos de relativa calma. De hecho, los portafolios de cobertura de las relaciones Olmeca-wTI y Olmeca-Brent observan valores máximos de 92.56\% (Tse y Tsui) y $91.04 \%$ (Engle), respectivamente. En este tenor, el modelo de Engle muestra el mejor desempeño predictivo cuando utilizamos el mercado de futuros del WTI como mecanismo para transferir el riesgo, excepto para la relación Maya-WTI, $64.91 \%$ contra $68.07 \%$. 
Gráfica 5. Dinámica de los índices eficientes de cobertura con el WTI, 2012-2013
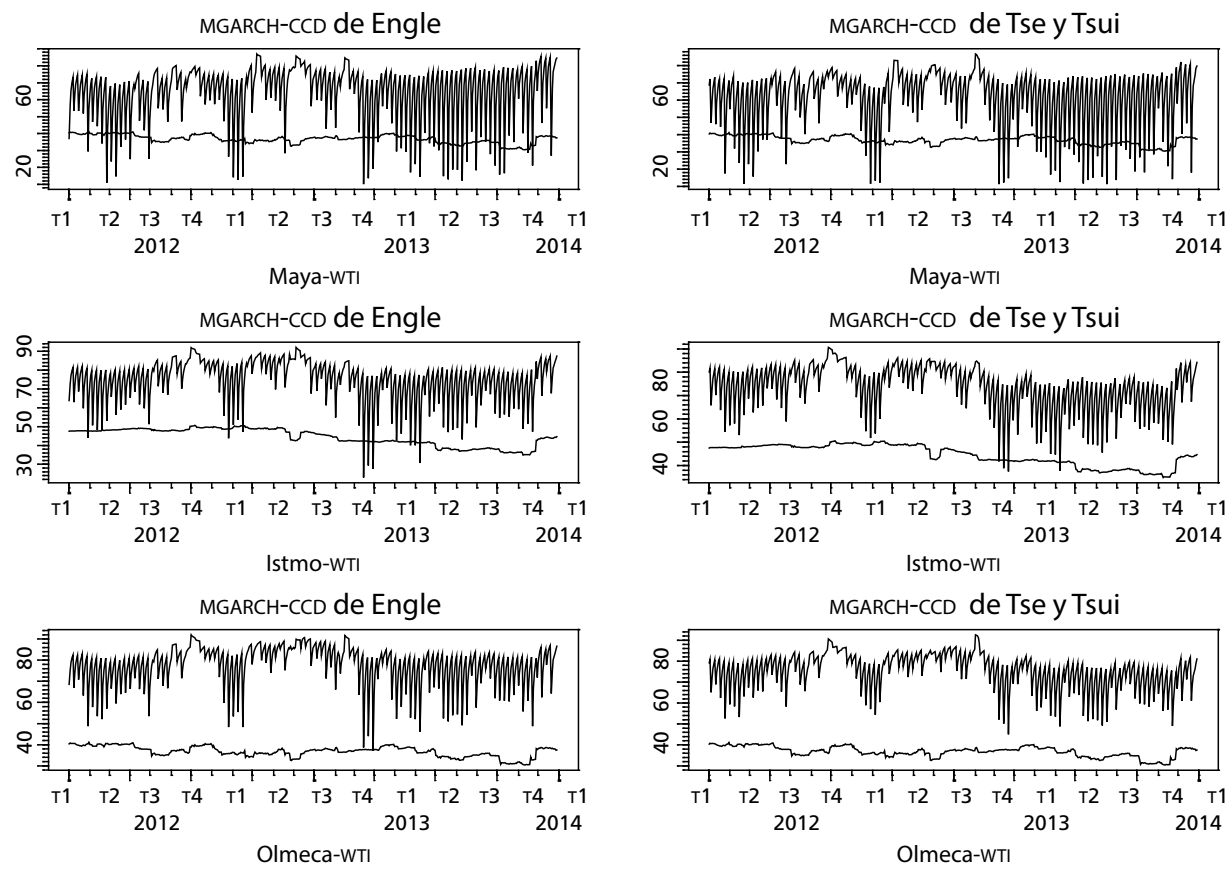

Fuente: Elaboración propia con información de la base de datos de Bloomberg.

Para la comparación de la eficiencia de la cobertura cruzada fuera de la muestra en el periodo del 3 de enero de 2012 al 31 de diciembre de 2013, el cuadro 6 reporta las varianzas de los portafolios de cobertura y los índices eficientes de cobertura que representan el promedio de la reducción del riesgo a través de los diferentes modelos y mercados petroleros. Para el de futuros del WTI, las estrategias de cobertura cruzada con MCO proporcionan a los inversionistas del mercado físico del petróleo Maya una reducción en el riesgo de $36.78 \%$ para una varianza de 1.3924, en promedio, en tanto que los participantes en los mercados del Istmo y el Olmeca alcanzan una reducción en el riesgo equivalente a 44.46\% y $62.11 \%$, con una varianza de 1.3472 y 0.8393 , respectivamente.

Resultados similares son obtenidos para el mercado de futuros del Brent, lo que hace evidente el pobre desempeño del modelo de MCO para prevenir el riesgo en el portafolio de cobertura con respecto a las coberturas cruzadas con MGARCH-CCD. En este contexto, el modelo MGARCH-CCD de Engle reduce el ries- 
Gráfica 6. Dinámica de los índices eficientes de cobertura con el Brent, 2012-2013
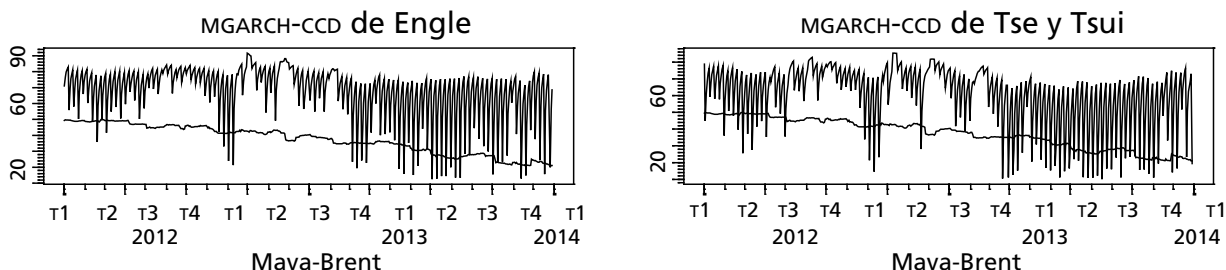

MGARCH-CCD de Engle

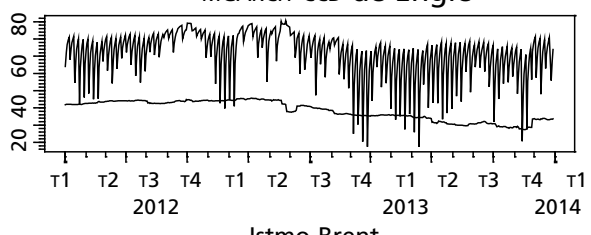

Istmo-Brent

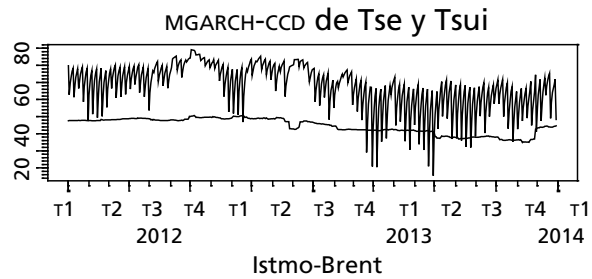

MGARCH-CCD de Engle
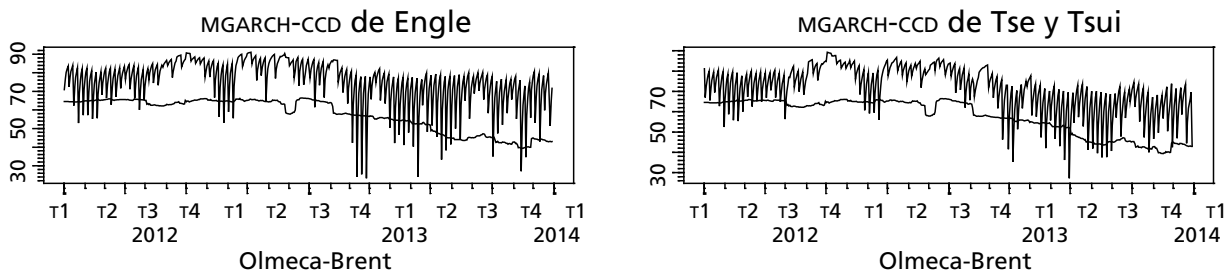

Fuente: Elaboración propia con información de la base de datos de Bloomberg.

\section{Cuadro 6. Eficiencia de la cobertura cruzada}

\begin{tabular}{|c|c|c|c|c|c|c|}
\hline \multirow[b]{2}{*}{ Modelo } & \multicolumn{3}{|c|}{$\begin{array}{c}\text { Varianza del portafolio de } \\
\text { cobertura }\end{array}$} & \multicolumn{3}{|c|}{ Reducción del riesgo } \\
\hline & Maya & Istmo & Olmeca & Maya & Istmo & Olmeca \\
\hline \multicolumn{7}{|l|}{ WTI } \\
\hline MCO & 1.3924 & 1.3472 & 0.8393 & 36.78 & 44.46 & 62.11 \\
\hline MGARCH-CCD de Engle & 1.4410 & 0.9585 & 0.7709 & 64.91 & 75.09 & 77.91 \\
\hline MGARCH-CCD de Tse y Tsui & 1.3699 & 0.8848 & 0.7631 & 62.06 & 73.96 & 75.62 \\
\hline \multicolumn{7}{|l|}{ Brent } \\
\hline MCO & 1.3576 & 1.4891 & 0.9301 & 37.48 & 38.49 & 57.63 \\
\hline MGARCH-CCD de Engle & 1.2488 & 1.0482 & 0.8028 & 68.07 & 71.62 & 75.53 \\
\hline MGARCH-CCD de Tse y Tsui & 1.3763 & 1.0778 & 0.8575 & 60.89 & 67.81 & 71.56 \\
\hline
\end{tabular}

Fuente: Elaboración propia con información de la base de datos de Bloomberg. 
go del portafolio hasta en $77.91 \%$ (varianza de 0.7709 ) para la relación OlmecaWTI, mientras que para las relaciones del WTI con el Istmo y el Maya los valores son de $75.09 \%$ (varianza de 0.9585 ) y $64.91 \%$ (varianza de 1.4410 ), respectivamente. Los resultados para las relaciones del Brent con el Olmeca, el Istmo y el Maya son muy similares: $75.53 \%$ (varianza de 0.8028 ), $71.62 \%$ (varianza de 1.0482 ) y $68.07 \%$ (varianza de 1.2488 ).

Analizando el desempeño del modelo MGARCH-CCD de Tse y Tsui en la reducción del riesgo en el portafolio, los resultados empíricos fuera de la muestra son superiores a los del modelo de MCO en ambos mercados de futuros. Sin embargo, los mejores resultados son alcanzados por el modelo de Engle: $77.91 \%$ contra $75.53 \%$ del modelo de Tse y Tsui para futuros de wTI en el caso del crudo Olmeca y $75.09 \%$ contra $71.62 \%$ para futuros del Brent respecto al Istmo. En esta circunstancia es importante destacar la calidad del petróleo nacional para alcanzar una mejor cobertura cruzada a un costo relativamente más bajo.

Para una justificación estadística de los resultados empíricos se analizó el comportamiento dinámico de las varianzas y covarianzas condicionales estimadas fuera de la muestra. ${ }^{12} \mathrm{El}$ análisis del mercado de futuros del WTI muestra que las varianzas condicionales del modelo de Engle son más volátiles y persistentes, aunque la covarianzas condicionales presentan la frecuencia de fluctuación más alta con respecto a los segundos momentos condicionales en ambos modelos MGARCH-CCD respecto al contrato de futuros del Brent. Estos hallazgos son también sustentados por la alta liquidez del mercado de futuros del WTI, la cual es medida por el creciente interés abierto y los excesivos volúmenes de negociación. Estos factores lo hacen más atractivo y eficiente en el diseño de estrategias de cobertura cruzada para que los productores y los consumidores de petróleo nacional puedan prevenir riesgos.

\section{Conclusiones}

El propósito de este trabajo es introducir términos de corrección de error en las ecuaciones de las medias condicionales de dos modelos de correlación condicional dinámica tradicionales. La nueva reparametrización es de gran utilidad para el diseño de coberturas cruzadas dinámicas de varianza mínima y con la ayuda

\footnotetext{
12 Por falta de espacio no se reportan las gráficas de las varianzas y covarianzas condicionales estimadas a través de los modelos MGARCH-CCD, pero están disponibles para cualquier aclaración de los resultados.
} 
de los mercados de futuros de los petróleos wTI y Brent como mecanismo para transferir la exposición al riesgo de los participantes en los mercados del petróleo mexicano. En el análisis fuera de la muestra, las RCCO estimadas por los modelos MGARCH-CCD presentan un comportamiento similar al de un proceso no estacionario para cada par de mercados, a diferencia de las del modelo de MCO. Esto es, regímenes de frecuencia baja y alta, derivados en gran medida de la evolución dinámica de las varianzas y covarianzas condicionales y las desviaciones de corto plazo entre los precios de contado y futuros del petróleo. Este hallazgo es relevante para los operadores en el mercado del petróleo mexicano, porque el uso de modelos de volatilidad multivariados les permite ajustar apropiadamente sus estrategias de cobertura cruzada de acuerdo con sus necesidades y las condiciones prevalecientes en el mercado. Con respecto al desempeño de los modelos MGARCH-CCD en la eficiencia de la cobertura fuera de la muestra, los resultados empíricos muestran que la administración de la exposición al riesgo en los mercados del petróleo mexicano es relativamente buena.

Esto se atribuye a que las estrategias de cobertura cruzada alcanzan a reducir el riesgo del portafolio, respecto al wTI y el Brent, hasta en $77.91 \%$ y $75.53 \%$, para el crudo Olmeca; en $75.09 \%$ y $71.62 \%$, para el Istmo, y en $64.91 \%$ y $68.07 \%$, para el Maya, mediante el modelo MGARCH-CCD de Engle, cuando la efectividad de cobertura máxima del modelo de MCo, en el caso del Olmeca, es de $62.11 \%$ y $57.63 \%$, respectivamente. Finalmente, aunque la cobertura cruzada óptima no es posible en ambos mercados de futuros, los hallazgos empíricos tienen importantes implicaciones económico-financieras para los consumidores $\mathrm{y}$, sobre todo, para las autoridades gubernamentales. El diseño de estrategias de cobertura cruzada más eficientes les permitirá transferir el riesgo de los movimientos adversos en los precios del petróleo mexicano y, al mismo tiempo, fortalecer las finanzas públicas y la competitividad económica del país. Asimismo, este tipo de operaciones serán más transparentes y con bajos costos de transacción, a diferencia de las coberturas en los mercados OTC, que se caracterizan por tener una estructura más peligrosa, agudizada por el riesgo de incumplimiento de las contrapartes.

\section{REFERENCIAS BIBLIOGRÁFICAS}

Alizadeh, Amir; Kavussanos, Manolis, y Menachof, David (2004), "Hedging against bunker price fluctuations using petroleum futures contract: constant versus time-varying hedge ratios", Applied Economic, 36 (12), pp. 1337-1353. 
Assis, Andre (2013), “An Investigation of Some Hedging Strategies for Crude Oil Market”, International Journal of Energy Economics and Policy, 3 (1), pp. 51-59.

Baillie, Richard, y Myers, Robert (1991), "Bivariate GARCH estimation of the optimal commodity futures hedge", Journal of Applied Econometrics, 6 (2), pp. 109-124.

Benet, Bruce (1992), "Hedge period length and ex ante futures hedging effectiveness: the case of foreign exchange rate cross hedges", Journal of Futures Markets, 12 (2), pp. 163-175.

Chang, Chia-Lin; McAleer, Michael, y Tansuchat, Roengchai (2009), "Modeling conditional correlations for risk diversification in crude oil markets", Journal of Energy Markets, 2 (4), pp. 29-51.

- (2010), "Analyzing and forecasting volatility spillovers, asymmetries and hedging in major oil markets", Energy Economics, 32 (6), pp. 1445-1455.

- (2011), "Crude oil hedging strategies using dynamic multivariate GARCH", Energy Economics, 33 (5), pp. 912-923.

Cecchetti, Stephen; Cumby, Robert, y Figlewski, Stephen (1988), "Estimation of the optimal futures hedge", The Review of Economics and Statistics, 70 (4), pp. 623-630.

Choudhry, Taufiq (2009), "Short-run deviations and time-varying hedge ratios: evidence from agricultural futures markets", International Review of Financial Analysis, 18 (1-2), pp. 58-65.

Daniel, James (2001), “Hedging government oil price risk”, IMF Working Paper 01/185.

Dávila, Javier; Núñez, José Antonio, y Ruiz, Antonio (2006), "Volatilidad del precio de la mezcla mexicana de exportación”, Economía: Teoría y Práctica, 0 (25), pp. 37-57.

Ederington, Louis (1979), "The hedging performance of the new futures markets", Journal of Finance, 34 (1), pp. 157-170.

Engle, Robert (2002), "Dynamic conditional correlation: a new simple class of multivariate GARCH models", Journal of Business and Economics Statistics, 20 (3), pp. 339-350.

Engle, Robert, y Granger, Clive (1987), "Co-integration and error correction: representation, estimation and testing", Econometrica, 55 (2), pp. 251-276.

Engle, Robert, y Kroner, Kenneth (1995), "Multivariate simultaneous generalized ARCH", Econometric Theory, 11 (1), pp. 125-150.

Figlewski, Stephen (1984), "Hedging performance and basis risk in stock index futures", Journal of Finance, 39 (3), pp. 657-669.

Geman, Hélyette, y Kharoubi, Cécile (2008), “WTI crude oil futures in portfolio diversifi- 
cation: The time-to-maturity effect", Journal of Banking and Finance, 32 (12), pp. 2553-2559.

Haigh, Michael, y Holt, Mattew (2002), "Crack spread hedging: accounting for timevarying spillovers in the energy futures markets", Journal of Applied Econometrics, 17 (3), pp. 269-289.

Hansen, Peter (2005), "A test for superior predictive ability", Journal of Business and Economics Statistics, 34 (4), pp. 365-380.

Hung, Jui; Wang, Yi; Chang, Matthew; Shih, Kuang, y Kao, Hsiu (2011), "Minimum variance hedging with bivariate regime-switching model for WTI crude oil", Energy, 36 (5), pp. 3050-3057.

Ku, Yuan-hun Hsu; Chen, Ho-chyhuan, y Chen, Kuang-hua (2007), "On the application of dynamic conditional correlation model in the estimating optimal timevarying hedge ratios", Applied Economics Letters, 14 (7), pp. 503-509.

Jalali, Ahmad, y Kazemi, Maryam (2006), "Price volatility, hedging and variable risk premium in the crude oil market", OPEC Review, 30 (2), pp. 55-70.

Jesús, Raúl de (2014), “Análisis del grado de integración de México en los mercados internacionales del petróleo basado en la dinámica de las correlaciones", trabajo de investigación, Facultad de Economía, UAEM.

Jesús, Raúl de, y Carvajal, Lidia (2013), "Modelación y predicción de la volatilidad con innovaciones de colas pesadas: evidencia empírica para los petróleos Maya y mezcla mexicana de exportación", Paradigma Económico, 5 (1), pp. 67-105.

Kroner, Kenneth, y Sultan, Jahangir (1993), "Time-varying distribution and dynamic hedging with foreign currency and futures", Journal of Financial and Quantitative Analysis, 28 (4), pp. 535-551.

Lanza, Alessandro; Manera, Matteo, y McAleer, Michael (2006), "Modeling dynamic conditional correlations in WTI oil forward and futures returns", Finance Research Letters, 3 (2), pp. 114-132.

Lorenzo, Arturo; Durán, Rocío, y Armenta, Leticia (2012), "Conditional correlation between oil and stock markets returns: The case of Mexico", Revista Mexicana de Economía y Finanzas, 7 (1), pp. 49-63.

Manera, Mateo; McAleer, Michael, y Grasso, Margherita (2006), "Modelling timevarying conditional correlations in the volatility of Tapis oil spot and forward returns", Applied Financial Economics, 16 (7), pp. 525-533.

Moschini, Gian Carlo, y Myers, Robert (2002), "Testing for constant hedge ratios in commodity markets: a multivariate GARCH approach", Journal of Empirical Finance, 9 (5), pp. 589-603. 
Myers, Robert, y Thompson, Stanley (1989), “Generalized optimal hedge ratio estimation”, American Journal of Agricultural Economics, 71 (4), pp. 858-868.

Park, Tae, y Switzer, Lorne (1995), "Bivariate GARCH estimation of the optimal hedge ratios for stock index futures: a note", Journal of Futures Markets, 15 (1), pp. 61-67.

Ripple, Ronald, y Moosa, Imad (2007), "Hedging effectiveness and futures contract maturity: the case of NYMEX crude oil futures", Applied Financial Economics, 17 (9), pp. 683-689.

Salle, Andre de (2013), "An investigation of some hedging strategies for crude oil market”, International Journal of Energy Economics and Policy, 3 (1), pp. 51-59.

Toyoshima, Yuki, Nakaijima, Tadahiro, y Hamori, Shigeyuki (2013). "Crude oil hedging strategy: new evidence from the data of the financial crisis", Applied Financial Economics, vol. 23, núm. 12, pp. 1033-1041.

Tse, Y. K., y Tsui Albert (2002), “A multivariate generalized autoregressive conditional heteroscedasticity model with time-varying correlations", Journal of Business and Economics Statistics, 20 (3), pp. 351-362.

Wang, Yudong, y Wu, Chongfeng (2012), "Forecasting energy market volatility using GARCH models: Can multivariate models beat univariate models?", Energy Economics, 34 (6), pp. 2167-2181. 